\title{
Tupfel Long Fin Nacre Zebrafish
}

National Cancer Institute

\section{Source}

National Cancer Institute. Tupfel Long Fin Nacre Zebrafish. NCI Thesaurus. Code C79984.

A wild-type zebrafish line established from the breeding of T upfel long fin parents and the subsequent selection and inbreeding of fish lacking melanophore pigmentation. 